\title{
SECOND-ORDER ABSORBING BOUNDARY CONDITIONS FOR THE WAVE EQUATION IN A RECTANGULAR DOMAIN
}

\author{
DONGWOO SHEEN
}

\begin{abstract}
We study finite element methods for the wave equation in a rectangular domain with a second-order absorbing boundary condition imposed on the boundary. For this problem there seems to be no known finite element method, although many finite difference methods have been proposed. A thirdorder energy, however, will be introduced which will be utilized to reduce our original second-order problem to a first-order symmetric dissipative hyperbolic system. Then, for this first-order system a weak formulation will be given and continuous-time and discrete-time Galerkin procedures will be investigated. Error estimates will also be given.
\end{abstract}

\section{INTRODUCTION}

We shall study the problem

$$
\begin{aligned}
\mathscr{L} u \equiv u_{t t}-\Delta u & =0 & & \text { on } \Omega \times J, \\
u_{t t}+2 u_{t \nu}+u_{\nu \nu} & =0 & & \text { on } \Gamma \times J, \\
u(x, y, 0) & =\phi(x, y) & & \text { on } \Omega, \\
u_{t}(x, y, 0) & =\psi(x, y) & & \text { on } \Omega,
\end{aligned}
$$

where $\Omega=\{(x, y) \mid 0<x<1,0<y<1\}, \Gamma=\partial \Omega$, and $J=[0, T]$ for $T>0$, and $\nu$ and $\tau$ denote the unit outward normal and the unit tangential vectors on $\Gamma$. The subscripts $t, x, y, \nu$, and $\tau$ of the variables will be used to indicate the derivatives of the variables with respect to the subscripts. Let

$$
\Gamma=\Gamma_{0}^{x} \cup \Gamma_{1}^{x} \cup \Gamma_{0}^{y} \cup \Gamma_{1}^{y},
$$

where

$$
\Gamma_{i}^{\zeta}=\{(x, y) \in \Gamma \mid \zeta=i\}, \quad \zeta=x, y, \quad i=0,1 .
$$

The boundary condition (1.1.ii) is a form of a second-order absorbing boundary condition suggested by Higdon [8] and Keys [10] independently. A general form of the $N$ th-order condition is given by

$$
\left[\prod_{j=1}^{N}\left(\cos \theta_{j} \frac{\partial}{\partial t}+\frac{\partial}{\partial \nu}\right)\right] u=0 \quad \text { on } \Gamma \times J,
$$

Received by the editor October 28, 1991.

1991 Mathematics Subject Classification. Primary 65N30, 35L05.

Key words and phrases. Finite element method, absorbing boundary conditions, higher-order energy. 
where $\left|\theta_{j}\right|<\pi / 2, j=1, \ldots, N$. In the above boundary condition each factor perfectly annihilates wave components arriving at $\Gamma$ with incident angle $\theta_{j}$. If the solution of (1.1) is smooth enough, an application of equation (1.1.i) shows that the boundary condition (1.1.ii) is equivalent to

$$
\begin{array}{ll}
u_{t t}-u_{t x}-\frac{1}{2} u_{y y}=0 & \text { on } \Gamma_{0}^{x} \times J, \\
u_{t t}+u_{t x}-\frac{1}{2} u_{y y}=0 & \text { on } \Gamma_{1}^{x} \times J, \\
u_{t t}-u_{t y}-\frac{1}{2} u_{x x}=0 & \text { on } \Gamma_{0}^{y} \times J, \\
u_{t t}+u_{t y}-\frac{1}{2} u_{x x}=0 & \text { on } \Gamma_{1}^{y} \times J,
\end{array}
$$

which is given by Engquist and Majda [5], and earlier proposed by Claerbout [2]. Indeed, Engquist and Majda [6] and Bamberger, Joly, and Roberts [1] suggested modified forms of (1.2) to take into account corner instability. However, in this paper we shall use the boundary condition (1.2) or (1.1.ii) for simplicity. Although many finite difference schemes have been proposed for the secondorder absorbing boundary condition (see, for example, $[3,4,5,6,8,9,10]$ ), no finite element approach seems to be known. The main difficulty comes from the order of the boundary condition for which it is not easy to derive a weak formulation which provides a consistent energy estimate with a suitable choice of a test function in the weak formulation. Recently, HaDuong and Joly introduced higher-order energies [7], which will turn out to be useful to initiate the study of a finite element approach to problem (1.1). HaDuong and Joly actually used a second-order energy for problem (1.1) in a half-plane to show stability of the problem. However, we shall see that if the domain is rectangular, the corresponding energy should be of third-order. Using this thirdorder energy, we are able to reduce problem (1.1) to a first-order symmetric dissipative hyperbolic system, for which finite element methods can be applied without difficulty.

The plan of this paper is as follows. We shall introduce a third-order energy associated with the system (1.1) in the next section. In $\S 3$ we shall derive a firstorder symmetric dissipative hyperbolic system from (1.1) using the third-order energy defined in $\S 2$; the first-order hyperbolic system will be analyzed and a weak formulation will be given. In the following $\S \S 4$ and 5 , continuous-time and discontinuous-time Galerkin procedures will be studied for the first-order hyperbolic system. Error estimates will be obtained for these schemes.

\section{HIGHER-ORDER ENERGY}

In this section we shall use the original idea of second-order energy introduced by HaDuong and Joly [7] to define a third-order energy associated with our problem (1.1). See also [14].

To begin, first observe that we can differentiate the equations (1.1.ii) on $\Gamma_{0}^{x} \cup \Gamma_{1}^{x}$ with respect to the $t$ - and $y$-variables to get

$$
\begin{array}{lll}
u_{t t t}-2 u_{t t x}+u_{t x x}=0, & u_{t t y}-2 u_{t x y}+u_{x x y}=0 & \text { on } \Gamma_{0}^{x}, \\
u_{t t t}+2 u_{t t x}+u_{t x x}=0, & u_{t t y}+2 u_{t x y}+u_{x x y}=0 & \text { on } \Gamma_{1}^{x} .
\end{array}
$$

Similarly, on $\Gamma_{0}^{y} \cup \Gamma_{1}^{y}$, by differentiating with respect to the $t$ - and $x$-variables,

$$
\begin{array}{lll}
u_{t t t}-2 u_{t t y}+u_{t y y}=0, & u_{t t x}-2 u_{t x y}+u_{x y y}=0 & \text { on } \Gamma_{0}^{y} \\
u_{t t t}+2 u_{t t y}+u_{t y y}=0, & u_{t t x}+2 u_{t x y}+u_{x y y}=0 & \text { on } \Gamma_{1}^{y} .
\end{array}
$$


Throughout this work, $(\cdot, \cdot)$ denotes the $L^{2}$-inner product over $L^{2}(\Omega)$ and $\langle\cdot, \cdot\rangle_{\Gamma}$ the $L^{2}$-inner product over the trace functions in $H^{1}(\Omega)$ on $\Gamma$. The corresponding norms will be denoted by $\|\cdot\|$ and $|\cdot|_{\Gamma}$, respectively. Also, for a nonnegative integer $k, H^{k}(\Omega)$ and $\|\cdot\|_{k}$ will be used to designate the standard Sobolev space and its norm.

In order to derive an energy identity, we recall that in the half-plane case HaDuong and Joly essentially added the $L^{2}$-inner product of the $t$-derivatives of $\mathscr{L} u$ and $u_{t}$ to the $L^{2}$-inner product of the $x$-derivatives of $\mathscr{L} u$ and $u_{t}$. The $x$-direction in this case was the normal direction to the artificial boundary of the half-plane. Therefore, in the rectangular domain case we consider the following $L^{2}$-inner product:

$$
0=\left(\left(\begin{array}{cc}
\frac{\partial^{2}}{\partial t^{2}} \mathscr{L} u & \frac{\partial^{2}}{\partial t \partial x} \mathscr{L} u \\
\frac{\partial^{2}}{\partial t \partial y} \mathscr{L} u & \frac{\partial^{2}}{\partial x \partial y} \mathscr{L} u
\end{array}\right),\left(\begin{array}{cc}
\frac{\partial^{2}}{\partial t^{2}} u_{t} & \frac{\partial^{2}}{\partial t \partial x} u_{t} \\
\frac{\partial^{2}}{\partial t \partial y} u_{t} & \frac{\partial^{2}}{\partial x \partial y} u_{t}
\end{array}\right)\right),
$$

for $u$ satisfying (1.1). By the divergence theorem and the conditions (2.1) on the boundary $\Gamma$, one can get

$$
\begin{gathered}
0=\frac{d}{d t} \frac{1}{2}\left\{\left(u_{t t t}, u_{t t t}\right)+\left(\nabla u_{t t}, \nabla u_{t t}\right)+\left(u_{t t x}, u_{t t x}\right)+\left(\nabla u_{t x}, \nabla u_{t x}\right)\right. \\
\left.+\left(u_{t t y}, u_{t t y}\right)+\left(\nabla u_{t y}, \nabla u_{t y}\right)+\left(u_{t x y}, u_{t x y}\right)+\left(\nabla u_{x y}, \nabla u_{x y}\right)\right\} \\
+2\left\{\left\langle u_{t t x}, u_{t t x}\right\rangle_{\Gamma_{0}^{x} \cup \Gamma_{1}^{x}}+\left\langle u_{t x y}, u_{t x y}\right\rangle_{\Gamma_{0}^{x} \cup \Gamma_{1}^{x}}\right. \\
\left.+\left\langle u_{t t y}, u_{t t y}\right\rangle_{\Gamma_{0}^{y} \cup \Gamma_{1}^{y}}+\left\langle u_{t x y}, u_{t x y}\right\rangle_{\Gamma_{0}^{y} \cup \Gamma_{1}^{y}}\right\} .
\end{gathered}
$$

Definition 2.1. A third-order energy $E_{\Omega}(u ; t)$ at time $t$ for the system (1.1) is a positive quadratic functional in the third-order derivatives of $u$ given by

$$
\begin{aligned}
E_{\Omega}(u ; t)=\frac{1}{2}\left\{\left(u_{t t t}, u_{t t t}\right)+\left(\nabla u_{t t}, \nabla u_{t t}\right)+\left(u_{t t x}, u_{t t x}\right)\right. \\
+\left(\nabla u_{t x}, \nabla u_{t x}\right)+\left(u_{t t y}, u_{t t y}\right)+\left(\nabla u_{t t y}, \nabla u_{t y}\right) \\
\left.+\left(u_{t x y}, u_{t x y}\right)+\left(\nabla u_{x y}, \nabla u_{x y}\right)\right\} .
\end{aligned}
$$

From (2.3) it follows that, for $0 \leq s \leq t$,

$$
E_{\Omega}(u ; t)-E_{\Omega}(u ; s)=-\int_{s}^{t} E_{\Gamma}(u ; r) d r,
$$

where

$$
E_{\Gamma}(u ; t)=2\left\{\left\langle u_{t t \nu}, u_{t t \nu}\right\rangle_{\Gamma}+\left\langle u_{t \nu \tau}, u_{t \nu \tau}\right\rangle_{\Gamma}\right\} .
$$

With the aid of (1.1.i), we can replace terms in (2.4) and (2.6) which contain more than two $t$-derivatives as follows:

$$
\begin{aligned}
E_{\Omega}(u ; t)=\frac{1}{2}\left\{2\left(u_{t x x}, u_{t x x}\right)\right. & +2\left(u_{t x x}, u_{t y y}\right)+2\left(u_{t y y}, u_{t y y}\right)+3\left(u_{t x y}, u_{t x y}\right) \\
+ & 2\left(u_{x x x}, u_{x x x}\right)+3\left(u_{x x y}, u_{x x y}\right)+3\left(u_{x y y}, u_{x y y}\right) \\
+ & \left.+2\left(u_{y y y}, u_{y y y}\right)+4\left(u_{x x x}, u_{x y y}\right)+4\left(u_{x x y}, u_{y y y}\right)\right\},
\end{aligned}
$$

$$
\begin{aligned}
E_{\Gamma}(u ; t)=2\left\{\left\langle u_{\nu \nu \nu}, u_{\nu \nu \nu}\right\rangle_{\Gamma}\right. & +2\left\langle u_{\nu \nu \nu}, u_{\nu \tau \tau}\right\rangle_{\Gamma} \\
& \left.+\left\langle u_{\nu \tau \tau}, u_{\nu \tau \tau}\right\rangle_{\Gamma}+\left\langle u_{t \nu \tau}, u_{t \nu \tau}\right\rangle_{\Gamma}\right\} .
\end{aligned}
$$

We thus have 
Theorem 2.1. Let $u$ satisfy the equations (1.1.i) and (1.1.ii) with sufficient regularity. Then the energy identity (2.5) holds for all $0 \leq s \leq t$, and the energy $E_{\Omega}(u ; t)$ dissipates in time:

$$
E_{\Omega}(u ; t) \leq E_{\Omega}(u ; s), \quad 0 \leq s \leq t .
$$

The boundary energy $E_{\Gamma}(u ; t)$ corresponds to the absorption of energy through the boundary $\Gamma$.

\section{FIRST-ORDER SYSTEM}

In this section the energy $E_{\Omega}(u ; t)$ of the form (2.7) will be used to give a first-order system related to problem (1.1) and then the first-order system will be analyzed.

The idea is motivated by representing $\frac{d}{d t} E_{\Omega}(u ; t)$ in the form

$$
\frac{d}{d t} E_{\Omega}(u ; t)=\left(A \underline{u}_{t}, \underline{u}\right),
$$

for a suitable positive-definite matrix $A$ and a variable $\underline{u}$. For this, write

$$
\underline{u}=\left(u_{t x x}, u_{t x y}, u_{t y y}, u_{x x x}, u_{x x y}, u_{x y y}, u_{y y y}\right)^{T} ;
$$

then such a matrix satisfying (3.1) is easily found:

$$
A=\left[\begin{array}{lll|llll}
2 & 0 & 1 & & & & \\
0 & 3 & 0 & & & 0 & \\
1 & 0 & 2 & & & & \\
\hline & & & 2 & 0 & 2 & 0 \\
& 0 & & 0 & 3 & 0 & 2 \\
& & & 0 & 3 & 0 \\
& & & 0 & 2 & 0 & 2
\end{array}\right] .
$$

In order to construct a first-order system, we use the wave equation (1.1.i) to get the following relations for $A \underline{u}_{t}$ :

$$
\begin{aligned}
& \frac{\partial}{\partial t}\left(2 u_{t x x}+u_{t y y}\right)=\frac{\partial}{\partial x}\left(2 u_{x x x}+2 u_{x y y}\right)+\frac{\partial}{\partial y}\left(u_{x x y}+u_{y y y}\right), \\
& \frac{\partial}{\partial t}\left(3 u_{t x y}\right)=\frac{\partial}{\partial x}\left(2 u_{x x y}+u_{y y y}\right)+\frac{\partial}{\partial y}\left(u_{x x x}+2 u_{x y y}\right), \\
& \frac{\partial}{\partial t}\left(u_{t x x}+2 u_{t y y}\right)=\frac{\partial}{\partial x}\left(u_{x x x}+u_{x y y}\right)+\frac{\partial}{\partial y}\left(2 u_{x x y}+2 u_{y y y}\right), \\
& \frac{\partial}{\partial t}\left(2 u_{x x x}+2 u_{x y y}\right)=\frac{\partial}{\partial x}\left(2 u_{t x x}+u_{t y y}\right)+\frac{\partial}{\partial y}\left(u_{t x y}\right), \\
& \frac{\partial}{\partial t}\left(3 u_{x x y}+2 u_{y y y}\right)=\frac{\partial}{\partial x}\left(2 u_{t x y}\right)+\frac{\partial}{\partial y}\left(u_{t x x}+2 u_{t y y}\right), \\
& \frac{\partial}{\partial t}\left(2 u_{x x x}+3 u_{x y y}\right)=\frac{\partial}{\partial x}\left(2 u_{t x x}+u_{t y y}\right)+\frac{\partial}{\partial y}\left(2 u_{t x y}\right), \\
& \frac{\partial}{\partial t}\left(2 u_{x x y}+2 u_{y y y}\right)=\frac{\partial}{\partial x}\left(u_{t x y}\right)+\frac{\partial}{\partial y}\left(u_{t x x}+2 u_{t y y}\right) .
\end{aligned}
$$

These relations (3.3) can be written in matrix form as

$$
A \underline{u}_{t}=B \underline{u}_{x}+C \underline{u}_{y} \quad \text { on } \Omega \times J,
$$


where

$$
B=\left[\begin{array}{lll|llll} 
& 0 & & 2 & 0 & 2 & 0 \\
& & & 1 & 2 & 0 & 1 \\
\hline 2 & 0 & 1 & & & & \\
0 & 2 & 0 & & 0 & \\
2 & 0 & 1 & & &
\end{array}\right], \quad C=\left[\begin{array}{lll|llll} 
& & 0 & 1 & 0 & 1 \\
& 0 & & 1 & 0 & 2 & 0 \\
& & 0 & 2 & 0 & 2 \\
\hline 0 & 1 & 0 & & & \\
1 & 0 & 2 & & 0 & \\
0 & 2 & 0 & & &
\end{array}\right] .
$$

Similarly, the boundary conditions $(2.1)$ can be expressed in matrix form as

$$
M_{i}^{\zeta} \underline{u}=0, \quad \Gamma_{i}^{\zeta} \times J, \quad \zeta=x, y, \quad i=0,1,
$$

where

$$
\begin{array}{rlrl}
M_{0}^{x} & =\left[\begin{array}{ccccccc}
2 & 0 & 1 & -2 & 0 & -2 & 0 \\
0 & -2 & 0 & 0 & 2 & 0 & 1
\end{array}\right], & & M_{1}^{x}=\left[\begin{array}{ccccccc}
2 & 0 & 1 & 2 & 0 & 2 & 0 \\
0 & 2 & 0 & 0 & 2 & 0 & 1
\end{array}\right], \\
M_{0}^{y}=\left[\begin{array}{ccccccc}
1 & 0 & 2 & 0 & -2 & 0 & -2 \\
0 & -2 & 0 & 1 & 0 & 2 & 0
\end{array}\right], & M_{1}^{y}=\left[\begin{array}{lllllll}
1 & 0 & 2 & 0 & 2 & 0 & 2 \\
0 & 2 & 0 & 1 & 0 & 2 & 0
\end{array}\right] .
\end{array}
$$

From the initial condition (1.1.iii) and (1.1.iv),

$$
\left.\underline{u}\right|_{t=0}=\Phi_{0} \quad \text { on } \Omega \text {, }
$$

where

$$
\Phi_{0}=\left(\psi_{x x}, \psi_{x y}, \psi_{y y}, \varphi_{x x x}, \varphi_{x x y}, \varphi_{x y y}, \varphi_{y y y}\right)^{T} .
$$

If the solution $\underline{u}$ satisfying (3.4), (3.5), and (3.6) is known, then the solution $u$ of problem (1.1) can be found by solving an initial value problem as follows.

First step: find $u_{x x}$ and $u_{y y}$ by solving

$$
\begin{array}{ll}
\frac{d}{d t} u_{x x}(x, y, t)=u_{t x x}(x, y, t), & u_{x x}(x, y, 0)=\varphi_{x x}(x, y), \\
\frac{d}{d t} u_{y y}(x, y, t)=u_{t y y}(x, y, t), & u_{y y}(x, y, 0)=\varphi_{y y}(x, y) .
\end{array}
$$

Second step: find $u_{t}$ by solving

$$
\frac{d}{d t} u_{t}(x, y, t)=u_{x x}(x, y, t)+u_{y y}(x, y, t), \quad u_{t}(x, y, 0)=\psi(x, y) \text {. }
$$

Third step: find $u$ by solving

$$
\frac{d}{d t} u(x, y, t)=u_{t}(x, y, t), \quad u(x, y, 0)=\varphi(x, y) .
$$

In the following we shall show that the solution of (3.4), (3.5), and (3.6) is unique, which will imply that the problem $(1.1)$ is equivalent to problems (3.4-3.9) if the data are sufficiently regular.

Introduce a variable $\underline{v}(x, y, t) \in \mathbf{R}^{7}$ and write

$$
\underline{v}=\left(v_{1}, v_{2}, v_{3}, v_{4}, v_{5}, v_{6}, v_{7}\right)^{T} \text {. }
$$

We are now interested in the hyperbolic system

$$
\begin{array}{lll}
\text { (3.10.i) } & A \underline{v}_{t}=B \underline{v}_{x}+C \underline{v}_{y} & \text { on } \Omega \times J, \\
\text { (3.10.ii) } & M_{i}^{\zeta} \underline{v}=0 & \text { on } \Gamma \times J, \quad \zeta=x, y, i=0,1, \\
\text { (3.10.iii) } & \left.\underline{v}\right|_{t=0}=\Phi_{0} & \text { on } \Omega .
\end{array}
$$


We shall show that the system (3.10.i) and (3.10.ii) forms a dissipative system. For this, we define an energy $E_{\Omega}(\underline{v} ; t)$ associated with the system (3.10.i) and (3.10.ii) for each time $t$ :

$$
E_{\Omega}(\underline{v} ; t)=\frac{1}{2}(A \underline{v}(\cdot, \cdot, t), \underline{v}(\cdot, \cdot, t)) .
$$

Then we have, for every $\underline{v}$ satisfying (3.10.i) and (3.10.ii),

$$
\begin{aligned}
\frac{d}{d t} \frac{1}{2}(A \underline{v}, \underline{v}) & =\frac{1}{2} \int_{\Omega}\left\{\left(\underline{v}^{T} B \underline{v}\right)_{x}+\left(\underline{v}^{T} C \underline{v}\right)_{y}\right\} d \Omega \\
& =\frac{1}{2} \int_{\Gamma_{0}^{x} \cup \Gamma_{1}^{x}} \underline{v}^{T} B \underline{v} \nu_{x} d \Gamma+\frac{1}{2} \int_{\Gamma_{0}^{y} \cup \Gamma_{1}^{y}} \underline{v}^{T} C \underline{v} \nu_{y} d \Gamma \\
& =-2 \int_{\Gamma_{0}^{x} \cup \Gamma_{1}^{x}}\left\{\left(v_{4}+v_{6}\right)^{2}+v_{2}^{2}\right\} d \Gamma-2 \int_{\Gamma_{0}^{y} \cup \Gamma_{1}^{y}}\left\{\left(v_{5}+v_{7}\right)^{2}+v_{2}^{2}\right\} d \Gamma,
\end{aligned}
$$

where $\nu_{x}$ and $\nu_{y}$ designate the $x$ - and $y$-components of $\nu$. Therefore, by defining a boundary absorption energy $E_{\Gamma}(\underline{v} ; t)$ by

$$
E_{\Gamma}(\underline{v} ; t)=2 \int_{\Gamma_{0}^{x} \cup \Gamma_{1}^{x}}\left\{\left(v_{4}+v_{6}\right)^{2}+v_{2}^{2}\right\} d \Gamma+2 \int_{\Gamma_{0}^{y} \cup \Gamma_{1}^{y}}\left\{\left(v_{5}+v_{7}\right)^{2}+v_{2}^{2}\right\} d \Gamma
$$

we get

$$
\frac{d}{d t} E_{\Omega}(\underline{v} ; t)+E_{\Gamma}(\underline{v} ; t)=0 .
$$

By integrating in time over $[s, t]$, we obtain

$$
E_{\Omega}(\underline{v} ; t)-E_{\Omega}(\underline{v} ; s)=-\int_{s}^{t} E_{\Gamma}(\underline{v} ; r) d r .
$$

Since $E_{\Gamma}(\underline{v} ; r) \geq 0$, the energy dissipativity

$$
E_{\Omega}(\underline{v} ; t) \leq E_{\Omega}(\underline{v} ; s), \quad 0 \leq s \leq t,
$$

follows; therefore, the solution of (3.10) is unique.

We have thus proven

Theorem 3.1. Suppose that $\underline{v}$ satisfies (3.10.i) and (3.10.ii). Then the energy identity (3.14) holds. Moreover, the solution of (3.10) is unique.

By the uniqueness of Problem (3.10), if the solution of (1.1) exists and satisfies a certain regularity, this solution can be obtained by solving the problems (3.4-3.9).

For a formulation of a weak form of problem (3.10), consider the space

$$
W_{1}(\Omega)=\left\{\underline{w} \in\left[H^{1}(\Omega)\right]^{7} \mid M_{i}^{\zeta} \underline{w}=0, \Gamma_{i}^{\zeta}, \zeta=x, y, i=0,1\right\} .
$$

Then the weak formulation of the problem is to find a differentiable map $\underline{v}: J \rightarrow$ $W_{1}(\Omega)$ satisfying

$$
\begin{array}{ll}
\left(A \underline{v}_{t}-B \underline{v}_{x}-C \underline{v}_{y}, \underline{w}\right)=0, & \underline{w} \in W_{1}(\Omega), \\
(\underline{v}(0), \underline{w})=\left(\Phi_{0}, \underline{w}\right), & \underline{w} \in W_{1}(\Omega) .
\end{array}
$$


It can be shown by a similar transformation as in [14] that the boundary condition (3.10.ii) leads to a well-posed condition so that the symmetric hyperbolic problem (3.10) is strongly well posed. This also provides the existence of a solution to (3.16).

\section{The continuous-Time Galerkin PROCEDURe}

We shall study the continuous-time Galerkin procedure for the resulting firstorder system derived in the last section. For this, a more convenient first-order system will be derived.

By the change of variable $\underline{z}=A^{1 / 2} \underline{v}$, problem (3.10) can be equivalently given as

$$
\begin{array}{ll}
\underline{z}_{t}=\mathscr{P} \underline{z} & \text { on } \Omega \times J, \\
\mathscr{B} \underline{z}=0 & \text { on } \Gamma \times J, \\
\left.\underline{z}\right|_{t=0}=\Phi_{1} & \text { on } \Omega,
\end{array}
$$

where $J=[0, T]$ for $T>0$ and

$$
\begin{aligned}
& \mathscr{P} \underline{z}=B_{1} \underline{z}_{x}+C_{1} \underline{z}_{y}, \\
& \mathscr{B} \underline{z}=M_{i}^{\zeta} A^{-1 / 2} \underline{z} \quad \text { on } \Gamma_{i}^{\zeta}, \quad \zeta=x, y, \quad i=0,1, \\
& \Phi_{1}=A^{1 / 2} \Phi_{0},
\end{aligned}
$$

with $B_{1}=A^{-1 / 2} B A^{-1 / 2}, C_{1}=A^{-1 / 2} C A^{-1 / 2}$. We observe that for all $\underline{w}$ satisfying (4.1.ii)

$$
\left(B_{1} \underline{w}_{x}+C_{1} \underline{w}_{y}, \underline{w}\right) \leq 0 .
$$

Furthermore, notice that (4.1.i) is a symmetric hyperbolic system such that (4.1) is well posed. The boundary $\Gamma=\partial \Omega$ is uniformly characteristic. Indeed,

$$
\operatorname{rank}\left(B_{1} \nu_{1}(x, y)+C_{1} \nu_{2}(x, y)\right) \equiv 4 \leq 7, \quad(x, y) \in \Gamma .
$$

For the theory of well-posedness in the uniformly characteristic case, see [12, 13]. Such problems arise also in many physical phenomena, for instance, Maxwell's equations.

A modification of the subspace $W_{1}(\Omega)$ of $\left[H^{1}(\Omega)\right]^{7}$ is given by

$$
Z_{1}(\Omega)=\left\{\underline{w} \in\left[H^{1}(\Omega)\right]^{7} \mid M_{i}^{\zeta} A^{-1 / 2} \underline{w}=0 \text { on } \Gamma_{i}^{\zeta}, \zeta=x, y, i=0,1\right\} .
$$

Then a weak formulation of (4.1) is to find a differentiable map $\underline{z}: J \rightarrow Z_{1}(\Omega)$ such that

$$
\begin{array}{ll}
\left(\underline{z}_{t}-\mathscr{P}_{\underline{z}}, \underline{w}\right)=0, & \underline{w} \in Z_{1}(\Omega), \\
(\underline{z}(0), \underline{w})=\left(\Phi_{1}, \underline{w}\right), & \underline{w} \in Z_{1}(\Omega) .
\end{array}
$$

The following proposition is an easy consequence of dissipativity.

Proposition 4.1. Suppose that $\underline{z}(t) \in Z_{1}(\Omega)$ satisfies (4.3). Then

$$
\|\underline{z}(t)\| \leq\left\|\Phi_{1}\right\| \text {. }
$$

In order to discretize (4.3), let $\mathscr{T}_{h}$ be a quasi-regular partition of $\Omega$ into triangles or rectangles with diameter bounded by $h$. Let $k \geq 1$ be an integer, 
and let $0<h \leq 1$. Choose a standard finite element subspace $Z_{h}$ of $Z_{1}$ associated with $\mathscr{T}_{h}$ such that

$$
\inf _{\underline{\chi} \in Z_{h}}\left[\|\underline{v}-\underline{\chi}\|+h\|\underline{v}-\underline{\chi}\|_{1}\right] \leq \text { Const } h^{r}\|\underline{v}\|_{r}, \quad 1 \leq r \leq k,
$$

for all $\underline{v} \in H^{k}(\Omega) \cap Z_{1}(\Omega)$. Throughout the paper, Const will be used to denote generic constants.

By the continuous-time Galerkin approximation to the solution $\underline{z}$ of problem (4.3) we mean the differentiable map $\underline{U}: J \rightarrow Z_{h}$ satisfying

$$
\begin{aligned}
\left(\frac{\partial}{\partial t} \underline{U}-\mathscr{P} \underline{U}, \underline{w}\right) & =0, & & \underline{w} \in Z_{h}, \\
\left(\underline{U}(0)-\Phi_{1}, \underline{w}\right) & =0, & & \underline{w} \in Z_{h} .
\end{aligned}
$$

Let $N_{h}$ be the dimension of $Z_{h}$. If $\underline{U}_{j}, j=1, \ldots, N_{h}$, forms a basis for $Z_{h}$, then $\underline{U}$ can be put in the form

$$
\underline{U}(t)=\sum_{j=1}^{N_{h}} \alpha_{j}(t) \underline{U}_{j},
$$

where the $\alpha_{j}(t)$ are differentiable functions of $t$. By choosing $\underline{w}=\underline{U}_{j}, j=$ $1, \ldots, N_{h}$, in (4.6), an ordinary differential system for the $\alpha_{j}(t)$ is obtained. The initial approximation $\underline{U}(0)$ can be found, since the matrix $\left(\underline{U}_{i}, \underline{U}_{j}\right)_{i, j=1}^{N_{h}}$ is invertible (positive-definite). Also, $\|\underline{U}(0)\|$ is bounded by $\left\|\Phi_{1}\right\|$; indeed, from (4.6.ii) it follows that

$$
\|\underline{U}(0)\|^{2}=(\underline{U}(0), \underline{U}(0))=\left(\Phi_{1}, \underline{U}(0)\right) \leq\left\|\Phi_{1}\right\|\|\underline{U}(0)\| .
$$

The stability of the procedure (4.6) follows from Proposition 4.1, by replacing $\underline{z}$ by $\underline{U}$ in (4.4). An error estimate similar to that of Layton [11] can be derived as follows.

Theorem 4.2. Let $\underline{z}$ and $\underline{U}$ be the solutions of (4.1) and (4.6), respectively. Assume that $\underline{z}(t) \in H^{r+1}(\bar{\Omega})$ and $\underline{z}_{t}(t) \in H^{r+1}(\Omega), t \in J$, for some $r, 0 \leq$ $r \leq k-1$. Then there is a constant $C>0$ independent of $h, \Phi_{1}$, and $t$ that satisfies

$$
\begin{aligned}
\|\underline{z}(t)-\underline{U}(t)\| \leq \mathrm{Const}\left[h ^ { r + 1 } \left\{\left\|\Phi_{1}\right\|_{r+1}+\|\underline{z}(t)\|_{r+1}+\right.\right. & \left.\left\|\underline{z}_{t}\right\|_{L^{1}\left(0, t ; H^{r+1}(\Omega)\right)}\right\} \\
& \left.+h^{r}\|\underline{z}\|_{L^{1}\left(0, t ; H^{r}(\Omega)\right)}\right] .
\end{aligned}
$$

Proof. Let $\Pi$ be the $L^{2}(\Omega)$-orthogonal projection from $Z_{1}$ onto $Z_{h}$. Choose $\underline{w}(t)=\Pi \underline{z}(t)$ and set $\underline{\eta}(t)=\underline{z}(t)-\underline{w}(t), \underline{\zeta}(t)=\underline{U}(t)-\underline{w}(t)$. By $(4.5)$,

$$
\left\|\frac{\partial}{\partial t} \underline{\eta}(t)\right\|_{j} \leq \text { Const } h^{r-j}\left\|\frac{\partial}{\partial t} \underline{z}(t)\right\|_{r}, \quad j=0,1 .
$$

From (4.3.i) and (4.6.i), we have

$$
\left(\frac{\partial \underline{\zeta}}{\partial t}, \underline{v}\right)-(\mathscr{P} \underline{\zeta}, \underline{v})=\left(\underline{\eta}_{t}-\mathscr{P} \underline{\eta}, \underline{v}\right)
$$


for $\underline{v} \in Z_{h}$. By Proposition 4.1, (4.5), (4.8), and the triangle inequality,

$$
\begin{aligned}
\|\underline{\zeta}(t)\| \leq & \|\underline{\zeta}(0)\|+\int_{0}^{t}\left\|\underline{\eta}_{t}(s)-\mathscr{P} \underline{\eta}(s)\right\| d s \\
\leq & \|\underline{\zeta}(0)\|+\int_{0}^{t}\left\{\left\|\underline{\eta}_{t}(s)\right\|+\text { Const } \| \underline{\eta}_{1}\right\} d s \\
\leq & \|\underline{z}(0)-\underline{U}(0)\| \\
& + \text { Const }\left[h^{r+1}\|\underline{z}(0)\|_{r+1}+\int_{0}^{t}\left\{h^{r+1}\left\|\underline{z}_{t}(s)\right\|_{r+1}+h^{r}\|\underline{z}(s)\|_{r+1}\right\} d s\right] \\
\leq & \|\underline{z}(0)-\underline{U}(0)\| \\
& + \text { Const }\left[h^{r+1}\|\underline{z}(0)\|_{r+1}+h^{r+1} \int_{0}^{t}\left\|\underline{z}_{t}(s)\right\|_{r+1} d s+h^{r} \int_{0}^{t}\|\underline{z}(s)\|_{r+1} d s\right] .
\end{aligned}
$$

Hence, again by (4.5),

$$
\begin{aligned}
\|\underline{z}(t)-\underline{U}(t)\| \leq & \|\underline{\eta}(t)\|+\|\underline{\zeta}(t)\| \\
\leq & \|\underline{z}(0)-\underline{U}(0)\| \\
& +\operatorname{Const}\left[h^{r+1}\left\{\|\underline{z}(t)\|_{r+1}+\|\underline{z}(0)\|_{r+1}+\left\|\underline{z}_{t}\right\|_{L^{1}\left(0, t ; H^{r+1}(\Omega)\right)}\right\}\right. \\
& \left.+h^{r} \| \underline{z}_{L^{1}\left(0, t ; H^{r+1}(\Omega)\right)}\right] .
\end{aligned}
$$

According to (4.6.ii), for any $\underline{\chi} \in Z_{h}$,

$$
\|\underline{z}(0)-\underline{U}(0)\|^{2}=(\underline{z}(0)-\underline{U}(0), \underline{z}(0)-\underline{\chi}) \leq\|\underline{z}(0)-\underline{U}(0)\| \quad\|\underline{z}(0)-\underline{\chi}\|,
$$

which implies

$$
\|\underline{z}(0)-\underline{U}(0)\| \leq\|\underline{z}(0)-\underline{\chi}\| .
$$

Thanks to (4.5), taking the infimum of the last inequality over $\underline{\chi} \in W_{h}$ yields

$$
\|\underline{z}(0)-\underline{U}(0)\| \leq \text { Const } h^{r+1}\|\underline{z}(0)\|_{r+1} .
$$

The estimate (4.7) then follows by the combination of (4.9) and (4.10). This completes the proof.

\section{The DisCRETE-TIME GALERKIN PROCEDURE}

In this section we shall define the discrete-time Galerkin procedure and derive stability and suboptimal error estimates. Also, the existence of the solution will be proved. The scheme is stable independent of the choice of time step. This differs from the case of semibounded operators treated in [11, 15].

Let us introduce the following notations:

$$
g^{n+\frac{1}{2}}=\frac{g^{n}+g^{n+1}}{2}, \quad d_{t} g^{n}=\frac{g^{n+1}-g^{n}}{\Delta t}
$$

where $g^{n}(\cdot)=g(\cdot, n \Delta t)$ for a given time step $\Delta t$. Set $L=T / \Delta t$. The discretetime Galerkin approximation is then defined as a sequence $\left(\underline{U}^{n}\right)_{0 \leq n \leq L} \subset Z_{h}$ satisfying

$$
\begin{aligned}
\left(d_{t} \underline{U}^{n}, \underline{w}\right)-\left(\mathscr{P} \underline{U}^{n+\frac{1}{2}}, \underline{w}\right) & =0, & & \underline{w} \in Z_{h}, \quad 1 \leq n \leq L-1, \\
\left(\underline{U}^{0}-\Phi_{1}, \underline{w}\right) & =0, & & \underline{w} \in Z_{h} .
\end{aligned}
$$


If $\underline{U}_{j}, j=1, \cdots, N_{h}$, forms a basis for $W_{h}$ as before, $\left(\underline{U}^{n}\right)_{0 \leq n \leq L}$ can be expressed in the form $\underline{U}^{n}=\sum_{1}^{N_{h}} \alpha_{j}^{n} \underline{U}_{j}$ for some $\alpha_{j}^{n}$.

The above scheme is called the Crank-Nicolson-Galerkin scheme, and it is unconditionally stable. Indeed, from (5.1.i) we have that, if $\left(\underline{U}^{n}\right)_{0 \leq n \leq L}$ is the solution of $(5.1)$, then

$$
\left\|\underline{U}^{n}\right\| \leq\left\|\underline{U}^{0}\right\|
$$

for $n \geq 1$. As an immediate consequence of (5.2), the existence and uniqueness of the solution of the scheme (5.1) is established:

Theorem 5.1. There exists a unique solution $\left(\underline{U}^{n}\right)_{0 \leq n \leq L}$ of the scheme (5.1), regardless of the choice of $\Delta t>0$.

Proof. We know that $\alpha_{j}^{0}$ can be found by the argument immediately following the definition of the scheme (4.6). The uniqueness of the $\alpha_{j}^{n+1}$ for $n \geq 0$ follows at once from (5.2). Since the algebraic equations are linear, uniqueness implies the existence of the $\alpha_{j}^{n+1}$.

Note that our problem has a solution independent of the choice of the time stepsize $\Delta t$, while this is not generally true for the semibounded case [11, 15]. For the convergence of the scheme (5.1), we have the following theorem.

Theorem 5.2. The solution $\left(\underline{U}_{n}\right)_{0 \leq n \leq L}$ of the procedure (5.1) satisfies the following error estimate if the solution $\underline{u}$ of $(4.3)$ is sufficiently smooth:

$$
\max _{0 \leq n \leq L}\left\|\underline{z}^{n}-\underline{U}^{n}\right\|
$$

$$
\begin{aligned}
\leq \text { Const } & {\left[h^{r+1}\left\|\Phi_{0}\right\|_{r+1}+h^{r}\|\underline{z}\|_{L^{\infty}\left(0, T ; H^{r+1}(\Omega)\right)}\right.} \\
& \left.+h^{r+1}\left\|\frac{\partial \underline{z}}{\partial t}\right\|_{L^{2}\left(0, T ; H^{r+1}(\Omega)\right)}+(\Delta t)^{2}\left\|\frac{\partial^{3} \underline{z}}{\partial t^{3}}\right\|_{L^{2}\left(0, T ; L^{2}(\Omega)\right)}\right] .
\end{aligned}
$$

Proof. Let $\Pi, \underline{w}, \underline{\eta}, \underline{\zeta}$ be defined as in the proof of Theorem 4.2. Subtraction of the equation

$$
\left(d_{t} \underline{z}^{n}, \underline{w}\right)-\left(\mathscr{P} \underline{z}^{n+\frac{1}{2}}, \underline{w}\right)=\left(d_{t} \underline{z}^{n}-\frac{\partial}{\partial t} \underline{z}^{n+\frac{1}{2}}, \underline{w}\right)
$$

from (5.1) gives

$$
\left(d_{t} \underline{\zeta}^{n}, \underline{w}\right)-\left(\mathscr{P} \underline{\zeta}^{n+\frac{1}{2}}, \underline{w}\right)=\left(d_{t} \underline{\eta}^{n}-\mathscr{P} \underline{\eta}^{n+\frac{1}{2}}, \underline{w}\right)-\left(d_{t} \underline{z}^{n}-\frac{\partial}{\partial t} \underline{z}^{n+\frac{1}{2}}, \underline{w}\right) .
$$

Applying (5.2) for $\underline{U}^{n}=\underline{\zeta}^{n}$, one obtains

$$
\begin{aligned}
\left\|\underline{\zeta}^{n}\right\| & \leq\left\|\underline{\zeta}^{0}\right\|+\Delta t \sum_{n=0}^{L-1}\left\|d_{t} \underline{\eta}^{n}-\mathscr{P} \underline{\eta}^{n+\frac{1}{2}}-d_{t} \underline{z}^{n}+\frac{\partial}{\partial t} \underline{z}^{n+\frac{1}{2}}\right\| \\
& \leq\left\|\underline{\zeta}^{0}\right\|+\Delta t \sum_{n=0}^{L-1}\left\{\left\|d_{t} \underline{\eta}^{n}\right\|+\left\|\underline{\eta}^{n+\frac{1}{2}}\right\|_{1}+\left\|d_{t} \underline{z}^{n}-\frac{\partial}{\partial t} \underline{z}^{n+\frac{1}{2}}\right\|\right\} .
\end{aligned}
$$


But, by the Hölder inequality,

$$
\begin{aligned}
\left\|d_{t} \underline{\eta}^{n}\right\| & =\left\|\frac{1}{\Delta t} \int_{n \Delta t}^{(n+1) \Delta t} \frac{\partial \underline{\eta}}{\partial t}(s) d s\right\| \leq\left\|\frac{1}{\Delta t}\left[\int_{n \Delta t}^{(n+1) \Delta t}\left|\frac{\partial \underline{\eta}}{\partial t}(s)\right|^{2} d s\right]^{\frac{1}{2}}(\Delta t)^{\frac{1}{2}}\right\| \\
& =\frac{1}{(\Delta t)^{\frac{1}{2}}}\left[\int_{n \Delta t}^{(n+1) \Delta t}\left\|\frac{\partial \underline{\eta}}{\partial t}(s)\right\|^{2} d s\right]^{\frac{1}{2}},
\end{aligned}
$$

which implies, by (4.8),

$$
\begin{aligned}
\Delta t \sum_{n=0}^{L-1}\left\|d_{t} \underline{\eta}^{n}\right\| & \leq \sqrt{L}(\Delta t)^{\frac{1}{2}}\left[\int_{0}^{T}\left\|\frac{\partial \underline{\eta}}{\partial t}(s)\right\|^{2} d s\right]^{\frac{1}{2}}=T^{\frac{1}{2}}\left\|\frac{\partial \underline{\eta}}{\partial t}\right\|_{L^{2}\left(0, T ; L^{2}(\Omega)\right)} \\
& \leq \text { Const } T^{\frac{1}{2}} h^{r+1}\left\|\frac{\partial \underline{z}}{\partial t}\right\|_{L^{2}\left(0, T ; L^{r+1}(\Omega)\right)}
\end{aligned}
$$

Next,

$$
\begin{aligned}
\Delta t \sum_{n=0}^{L-1}\left\|\underline{\eta}^{n+\frac{1}{2}}\right\|_{1} & \leq \Delta t \sum_{n=0}^{L}\left\|\underline{\eta}^{n}\right\|_{1} \leq \Delta t \sum_{n=0}^{L} \operatorname{Const} h^{r}\left\|\underline{z}^{n}\right\|_{r+1} \\
& =\text { Const } T h^{r}\|\underline{z}\|_{L^{\infty}\left(0, T ; H^{r+1}(\Omega)\right)} .
\end{aligned}
$$

Now, by the Peano kernel theorem,

$$
\left|d_{t} \underline{z}^{n}-\frac{\partial}{\partial t} \underline{z}^{n+\frac{1}{2}}\right|=\frac{1}{2 \Delta t} \int_{n \Delta t}^{(n+1) \Delta t} \underline{z}_{t t t}(t)\{(n+1) \Delta t-t\}\{t-n \Delta t\} d t .
$$

By using (5.5), (5.6), and (5.7), $\left\|\underline{\zeta}^{n}\right\|$ can be estimated further as follows:

$$
\begin{aligned}
& \left\|\underline{\zeta}^{n}\right\| \leq\left\|\underline{\zeta}^{0}\right\|+\text { Const }\left[T^{\frac{1}{2}} h^{r+1}\left\|\frac{\partial \underline{z}}{\partial t}\right\|_{L^{2}\left(0, T ; L^{r+1}(\Omega)\right)}\right. \\
& \left.+T h^{r}\|\underline{z}\|_{L^{\infty}\left(0, T ; H^{r+1}(\Omega)\right)}+T^{\frac{1}{2}}(\Delta t)^{2}\left\|\underline{z}_{t t t}\right\|_{L^{2}\left(0, T ; L^{2}(\Omega)\right)}\right] .
\end{aligned}
$$

Therefore, again by (4.8),

$\left\|\underline{z}^{n}-\underline{U}^{n}\right\| \leq\left\|\underline{\zeta}^{n}\right\|+\left\|\underline{\eta}^{n}\right\|$

$$
\begin{aligned}
\leq \text { Const }\left[h^{r+1}\left\|\underline{z}^{n}\right\|_{r+1}+\left\|\underline{U}^{0}-\Pi \underline{z}^{0}\right\|+T^{\frac{1}{2}} h^{r+1}\left\|\frac{\partial \underline{z}}{\partial t}\right\|_{L^{r+1}\left(0, T ; L^{2}(\Omega)\right)}\right. \\
\left.+T h^{r}\|\underline{z}\|_{L^{\infty}\left(0, T ; H^{r+1}(\Omega)\right)}+T^{\frac{1}{2}}(\Delta t)^{2}\left\|\underline{z}_{t t t}\right\|_{L^{2}\left(0, T ; L^{2}(\Omega)\right)}\right] .
\end{aligned}
$$

Since

$$
\left\|\underline{U}^{0}-\Pi \underline{z^{0}}\right\| \leq\left\|\underline{U}^{0}-\underline{z}^{0}\right\|+\left\|\underline{z}^{0}-\Pi \underline{z}^{0}\right\| \leq\left\|\underline{U}^{0}-\underline{z}^{0}\right\|+h^{r+1}\left\|\underline{z}^{0}\right\|_{r+1},
$$

using the estimate (4.10), we arrive at (5.3).

\section{Conclusions}

A finite element approach has been established in order to treat second-order absorbing boundary conditions for wave propagations in a rectangular domain. 
This is based on the concept of a third-order energy, which generalizes that of the second-order energy of HaDuong and Joly. Once a good energy is obtained for a given problem, a decent first-order system can be obtained, for which standard Galerkin methods are applicable.

\section{ACKNOWLEDGMENT}

This work is based on a part of the author's doctoral dissertation at Purdue University. The author would like to thank his advisor, Professor Jim Douglas, Jr., for his help and support throughout the graduate study. He also wishes to acknowledge many suggestions of Professor Juan E. Santos.

\section{BIBLIOGRAPHY}

1. A. Bamberger, P. Joly, and J. E. Roberts, Second-order absorbing boundary conditions for the wave equation: A solution for the corner problem, SIAM J. Numer. Anal. 27 (1990), 323-352.

2. J. F. Claerbout, Fundamentals of geophysical data processing, McGraw-Hill, New York, 1976.

3. R. Clayton and B. Engquist, Absorbing boundary conditions for acoustic and elastic wave equations, Bull. Seismol. Soc. Amer. 67 (1977), 1529-1540.

4. S. H. Emerman and R. A. Stephen, Comment on "Absorbing boundary conditions for acoustic and elastic wave equations" by $R$. Clayton and B. Engquist, Bull. Seismol. Soc. Amer. 73 (1983), 661-665.

5. B. Engquist and A. Majda, Absorbing boundary conditions for the numerical simulation of waves, Math. Comp. 31 (1977), 629-651.

6. _ Radiation boundary conditions for acoustic and elastic wave calculations, Comm. Pure Appl. Math. 32 (1979), 313-357.

7. T. HaDuong and P. Joly, On the stability analysis of boundary conditions for the wave equation by energy methods, Part I: The homogeneous case, Report No. 224, Ecole Polytechnique, Centre de Mathématiques Appliquées, 1990.

8. R. L. Higdon, Absorbing boundary conditions for difference approximations to the multidimensional wave equation, Math. Comp. 47 (1986), 437-459.

9. __ Numerical absorbing boundary conditions for the wave equation, Math. Comp. 49 (1987), 65-90.

10. R. G. Keys, Absorbing boundary conditions for acoustic media, Geophys. 50 (1985), 892-902.

11. W. J. Layton, Stable Galerkin methods for hyperbolic systems, SIAM J. Numer. Anal. 20 (1983), 221-223.

12. A. Majda and S. Osher, Initial-boundary value problems for hyperbolic equations with uniformly characteristic boundary, Comm. Pure Appl. Math. 28 (1975), 607-675.

13. J. Rauch, Symmetric positive systems with boundary characteristic of constant multiplicity, Trans. Amer. Math. Soc. 291 (1985), 167-187.

14. D. Sheen, On the wave equation in a half plane with second order absorbing boundary conditions (to appear).

15. B. Swartz and B. Wendroff, Generalized finite-difference schemes, Math. Comp. 23 (1969), 37-49.

Department of Mathematics, Purdue University, West Lafayette, Indiana 47907

E-mail address: sheen@math.purdue.edu

Istituto di Analisi Numerica del CNR, Corso Carlo Alberto 5, 27100 Pavia, Italy

E-mail address: sheen@ipvcube.ian.pv.cnr.it

Current address: Department of Mathematics, Seoul National University, Seoul 151-742, Korea

E-mail address: sheen@math.snu.ac.kr 